\title{
Reversible growth arrest of 3D tumor spheroids stored in oxygen absorber-induced anoxia
}

\author{
AURÉLIE GOMES ${ }^{1}$, MATTHIEU DEFAUX ${ }^{2}$, RIWANON MICHEL LEMEE ${ }^{3}$, \\ VALÉRIE LOBJOIS $^{1}$ and BERNARD DUCOMMUN ${ }^{1,4}$
}

\begin{abstract}
${ }^{1}$ Institut des Technologies Avancées en Sciences du Vivant, Université de Toulouse and Centre National de la Recherche Scientifique, 31106 Toulouse; ${ }^{2}$ Atmosphère Contrôle SAS, 14760 Bretteville sur Odon; ${ }^{3}$ Laboratoires Standa, 14050 Caen; ${ }^{4}$ Oncology Department, Centre Hospitalier Universitaire de Toulouse, 31059 Toulouse, France
\end{abstract}

Received June 30, 2017; Accepted September 20, 2017

DOI: $10.3892 / \mathrm{ol} .2017 .7465$

\begin{abstract}
Multicellular tumor spheroids models are of increasing interest in preclinical studies and pharmacological evaluation. However, their storage and transport is often a limitation because it requires adapted and expensive procedures. Here, we propose a very simple method to store 3D spheroids, using a procedure based on oxygen absorber-induced anoxia. We report that oxygen absorbers allow generating an anoxic environment for spheroid storage in culture plates. Oxygen absorber-induced anoxia fully and reversibly arrests spheroid growth for 4 days at $37^{\circ} \mathrm{C}$ and up to 18 days at $4^{\circ} \mathrm{C}$. We then show that the response to etoposide is comparable in spheroids preserved in conditions of absorber-induced anoxia at $4^{\circ} \mathrm{C}$ and spheroids kept in normoxia at $37^{\circ} \mathrm{C}$. These results represent a major improvement that should simplify the storage, transport and use of 3D spheroids.
\end{abstract}

\section{Introduction}

To reproduce the three-dimensional (3D) organization and the cell-cell and cell-matrix features that are found in normal or tumor tissues, cells can be cultured as 3D aggregates, also called spheroids. Over the last decade, spheroids have been recognized as essential 3D culture models for high-throughput screening and pharmacological evaluation (1). They are also of utmost interest in the field of tissue engineering because they represent basic bricks that can be used to generate original cell assemblages and organization, and to produce larger tissues (2).

Correspondence to: Professor Bernard Ducommun or Dr Valérie Lobjois, Institut des Technologies Avancées en Sciences du Vivant, Université de Toulouse and Centre National de la Recherche Scientifique, 1 Place Pierre Potier, 31106 Toulouse, France E-mail: bernard.ducommun@itav.fr

E-mail: valerie.lobjois@itav.fr

Key words: 3D spheroids, oxygen absorber, anoxia, microtissue storage
Spheroids derived from primary cells or immortalized cancer cell lines are often made using the classical hanging drop or centrifugation methods $(3,4)$. Although these techniques are robust and their variability is acceptably low, there are potential reproducibility issues linked to various classical experimental problems and batch-to-batch variability. Storage in liquid nitrogen, in the presence of a cryoprotectant, has been used for brain cell and hepatocyte spheroids with successful preservation of morphological markers and functionality $(5,6)$. However, most researchers rely on repeated custom production, depending on the need. Alternatively, spheroids can be ordered from companies that deliver standardized and perfectly controlled biological material, usually shipped in conditions that maintain the microtissues at $37^{\circ} \mathrm{C}$ during transit. In such case, temperature stabilization and shipping efficiency become critical issues.

Several reports have shown that spheroid growth results in the generation of a hypoxia and nutrient gradients (7-9) where cells localized in the inner region exit the cell cycle and enter a G0 quiescent state. The induced cell quiescence in the spheroid central region mimics the situation observed in vivo in microtumor domains and the subsequent resistance to classical chemotherapeutic agents $(10,11)$. Moreover, we recently demonstrated that oxygen partial pressure is a rate-limiting parameter for cell proliferation in $3 \mathrm{D}$ spheroids (12). Thus, we hypothesized that culturing spheroids in anoxic conditions could be an efficient way to induce their reversible growth arrest. In order to make the procedure as simple and inexpensive as possible, we investigated whether oxygen absorbers could be used to generate an anoxic environment for microtissues. Oxygen absorbers can efficiently reduce oxygen concentration to less than $0.0001 \%$. Consequently, their composition and packaging has been adapted for a very large range of applications (13). Currently, oxygen absorbers are used to preserve many food products (bread, meat, fish and seafood, fruits, nuts, cheese) from food spoilage due to aerobic microorganism proliferation and to prevent fat oxidation. They are also used in the pharmaceutical industry to improve molecule protection and safety. Oxygen absorbers are also employed to generate oxygen-free environments to control and eradicate museum insect pest and to preserve art works $(14,15)$. 
Here, we report that in oxygen absorber-induced anoxia, spheroid growth can be reversibly stopped at $4^{\circ} \mathrm{C}$ for up to 18 days. Moreover, after anoxic storage, they can be successfully used in pharmacological assays. This oxygen absorber-based method to reversibly stop cell proliferation could represent a tremendous advance in the field of 3D microtissue engineering with obvious immediate applications for spheroid storage and shipment.

\section{Materials and methods}

Cell culture. HCT116 colon adenocarcinoma cells (ATCC) were cultured in DMEM (Invitrogen; Thermo Fisher Scientific, Inc., Waltham, MA, USA) containing $10 \%$ foetal calf serum (FCS), $2 \mathrm{mM} / 1$ glutamine and penicillin/streptomycin in a tissue culture incubator (humidified atmosphere of $5 \% \mathrm{CO}_{2}$ at $37^{\circ} \mathrm{C}$ ). Spheroids were prepared as previously described(9). Briefly, 500 cells/well were distributed in ultra-low attachment 96-round bottom well plates. After centrifugation at $200 \mathrm{~g}$ for $6 \mathrm{~min}$, plates were placed in the tissue culture incubator. After 3 days, each well contained a single spheroid. The spheroid maximal area was determined by automated measurement with the High Content Screening ArrayScan Cellomics ${ }^{\circledR}$ platform (Thermo Fischer Scientific Inc.).

Packaging with oxygen absorbers and oxygen concentration measurement. For anoxic storage, each 96-well plate was placed in an ATCO Biocult P plastic bag with or without (control) an $\mathrm{ATCO}^{\circledR}$ Biosystem 96P oxygen absorber (Fig. 1A). The bag was immediately heat-sealed and placed in the tissue culture incubator (storage at $37^{\circ} \mathrm{C}$ ) or in a cold room (storage at $4^{\circ} \mathrm{C}$ ). The $\mathrm{ATCO}^{\circledR}$ Biosystem $96 \mathrm{P}$ oxygen absorbers used in this study were specifically designed for 96-well plates. The residual oxygen concentration in the bag was determined using a Servomex 570A oximeter (accuracy in the range of $\pm 0.1 \%$; Servomex, Norwood, MA, USA). The oximeter probe was inserted in the sealed bag in an airtight manner and was positioned either outside or inside the 96-well plate. The residual oxygen level was calculated as the mean of at least three measurements at each time point. At the end of the storage period in anoxic conditions, bags were opened and plates returned to the tissue culture incubator.

Pharmacological evaluation. Spheroids were prepared in normoxia and then stored at $4^{\circ} \mathrm{C}$ in oxygen absorber-induced hypoxia ( $0 \%$ oxygen) for 4,7 or 14 days and then transferred to normoxia $\left(21 \%\right.$ oxygen at $37^{\circ} \mathrm{C}$ ) for $24 \mathrm{~h}$. This recovery time was chosen in order to use spheroids of approximately 350-400 $\mu \mathrm{m}$ in diameter to allow comparison with controls (spheroids of similar size cultured in normoxia). To test their response to etoposide, $100 \mu \mathrm{l}$ of culture medium per well was mixed with $100 \mu l$ of culture medium containing etoposide (Sigma-Aldrich, St. Louis, MO, USA). In each plate, six different concentrations were obtained by serial dilution (4-6 wells/spheroids for each concentration). Spheroid size was determined with the High Content Screening ArrayScan Cellomics ${ }^{\circledR}$ platform after $72 \mathrm{~h}$-incubation with etoposide. The half maximal inhibitory concentration $\left(\mathrm{IC}_{50}\right)$ was calculated using the Prism ${ }^{\circledR}$ software.
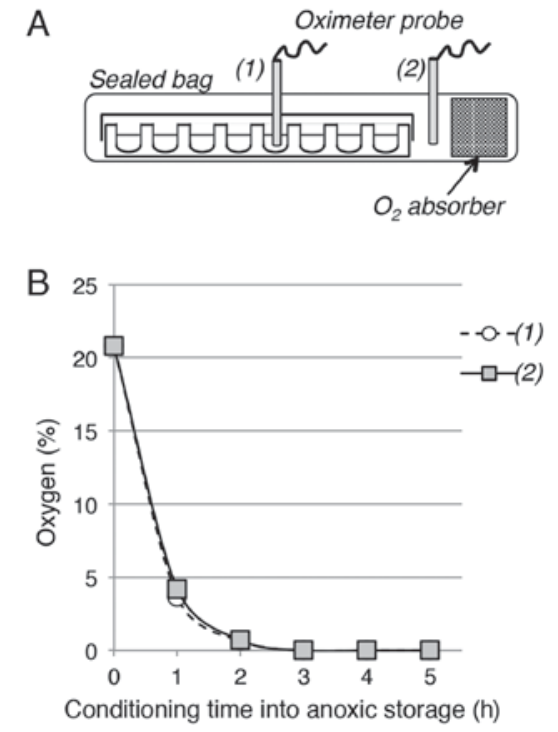

Figure 1. Oxygen absorbers to create an anoxic environment for 96-well plates. (A) Schematic representation of the experimental setting. The oxygen absorber ATCO Biosystem 96P is placed with a 96-well plate inside a plastic bag that is heat-sealed. The oximeter needle probe is positioned inside (1) or outside (2) the plate. (B) Residual oxygen concentration inside (1) and outside the plate (2) at different time points after the bag was sealed.

\section{Results and Discussion}

Use of an oxygen absorber to generate an anoxic environment for 96-well plates. Multi-well plates are the most commonly used cell culture disposable material to produce and transfer 3D spheroids. To analyse whether oxygen absorbers could be used to generate a hypoxic environment for spheroids cultured in 96-well plates, the oxygen concentration was measured with an oximeter needle probe positioned either on one side or in the middle of the 96-well plate packaged with an oxygen absorber in a heat-sealed plastic bag (Fig. 1A). The oxygen concentration measured inside the bag and in the plate rapidly decreased from $20.8 \%$ to $\sim 4 \%$ after $1 \mathrm{~h}$, to $0.7 \%$ after $2 \mathrm{~h}$ and nearly to $0 \%$ after $3 \mathrm{~h}$ (Fig. 1B). This indicates that this very simple experimental setting allows the fast and efficient removal of oxygen to generate an anoxic environment compatible with cell culture disposable material, such as 96 -well plates.

Oxygen absorber-induced anoxia reversibly arrests spheroid growth at $37^{\circ} \mathrm{C}$. The effect of absorber-induced anoxia on spheroid proliferation was then analysed with the experimental setting depicted in Fig. 2A that was used for all the experiments of this study. After 3 days in normoxia at $37^{\circ} \mathrm{C}$, each 96-well plate that contained one HCT116 colon adenocarcinoma spheroid per well was placed, with or without (control) one ATCO Biosystem 96P oxygen absorber, in a plastic bag that was heat-sealed and put back in the tissue culture incubator for 4 or 11 days. Visual inspection under an inverted microscope of spheroids after return to normoxic conditions (Fig. 2B, upper and lower panels, respectively) showed that control spheroids (no oxygen absorber) kept growing during storage and after removal from the bag. Conversely, growth of spheroids packed with the oxygen absorber was completely inhibited by anoxia. Upon return to normoxia, spheroid growth resumption was observed only after anoxic storage for 4 days, 
Table I. Determination of the $\mathrm{IC}_{50}$ for etoposide in spheroids stored in anoxic conditions.

\begin{tabular}{lcc}
\hline & \multicolumn{2}{c}{$\mathrm{IC}_{50}(\mu \mathrm{M})$} \\
\cline { 2 - 3 } Anoxic storage duration & Control & Anoxia at $4^{\circ} \mathrm{C}$ \\
\hline 4 days & 1.4 & 2.3 \\
7 days & 2.1 & 2.4 \\
14 days & 1.6 & 1.7 \\
\hline
\end{tabular}

Spheroids were prepared in normoxia and then stored at $4^{\circ} \mathrm{C}$ in oxygen absorber-induced hypoxia ( $0 \%$ oxygen) or in normoxia ( $21 \%$ oxygen at $37^{\circ} \mathrm{C}$; controls) for 4,7 or 14 days. Spheroid volume was determined at increasing concentratzions of etoposide (4 to 6 spheroids for each concentration). Curve fitting allowed calculating the $\mathrm{IC}_{50} . \mathrm{IC}_{50}$, half maximal inhibitory concentration.

A
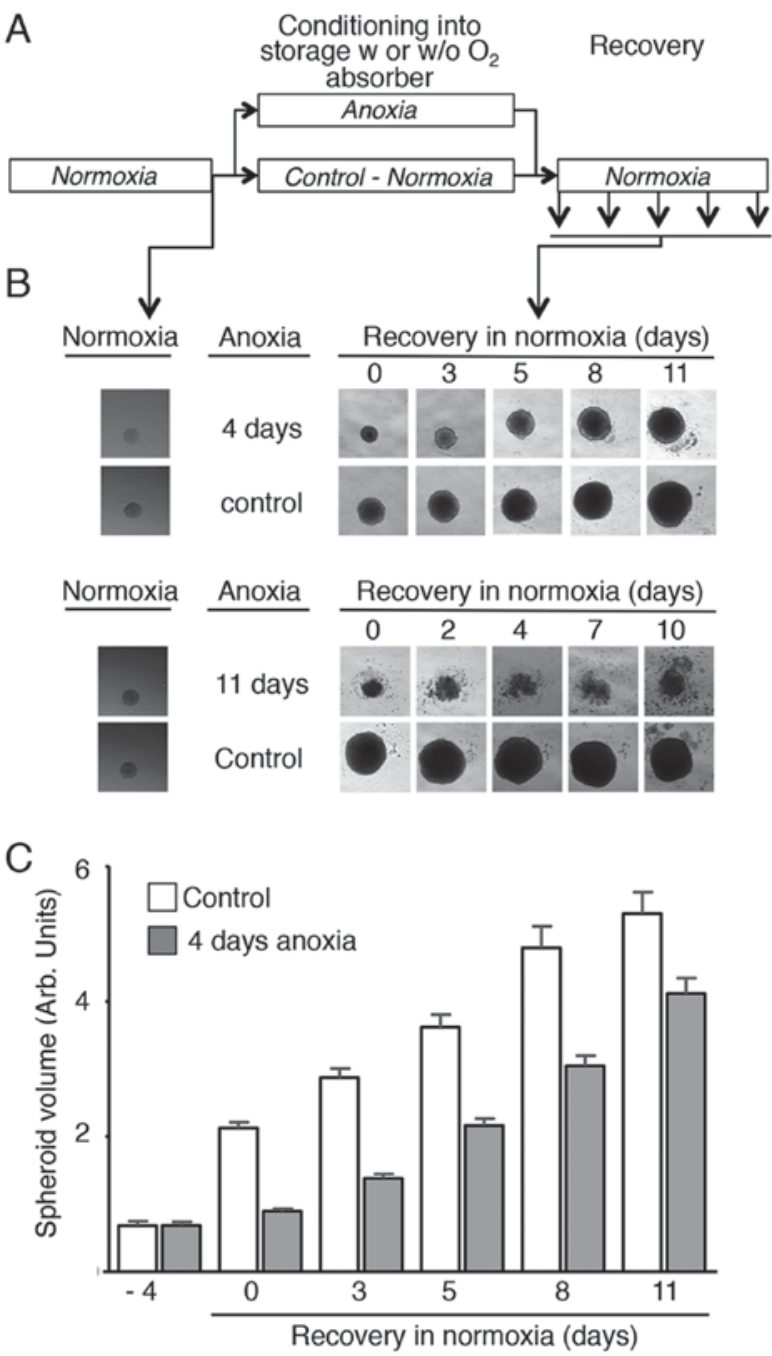

Figure 2. Reversible growth arrest after absorber-induced anoxia for 4 days at $37^{\circ} \mathrm{C}$. (A) Schematic representation of the experiments. Spheroids were prepared and grown initially in normoxia, then stored at $37^{\circ} \mathrm{C}$ in the presence or absence (control) of an oxygen absorber to generate hypoxia for 4 or 11 days. Then, spheroids were allowed to recover at $37^{\circ} \mathrm{C}$ in normoxia. (B) Representative micrographs illustrating the effect of anoxia at $37^{\circ} \mathrm{C}$ for 4 and 11 days on spheroid growth. (C) Spheroid volume determination before (-4), after 4 days of anoxia at $37^{\circ}(0)$ and during recovery. Data are the mean $( \pm \mathrm{SD})$ of two independent experiments with one plate of spheroid (i.e., 60 spheroids) for each time point.
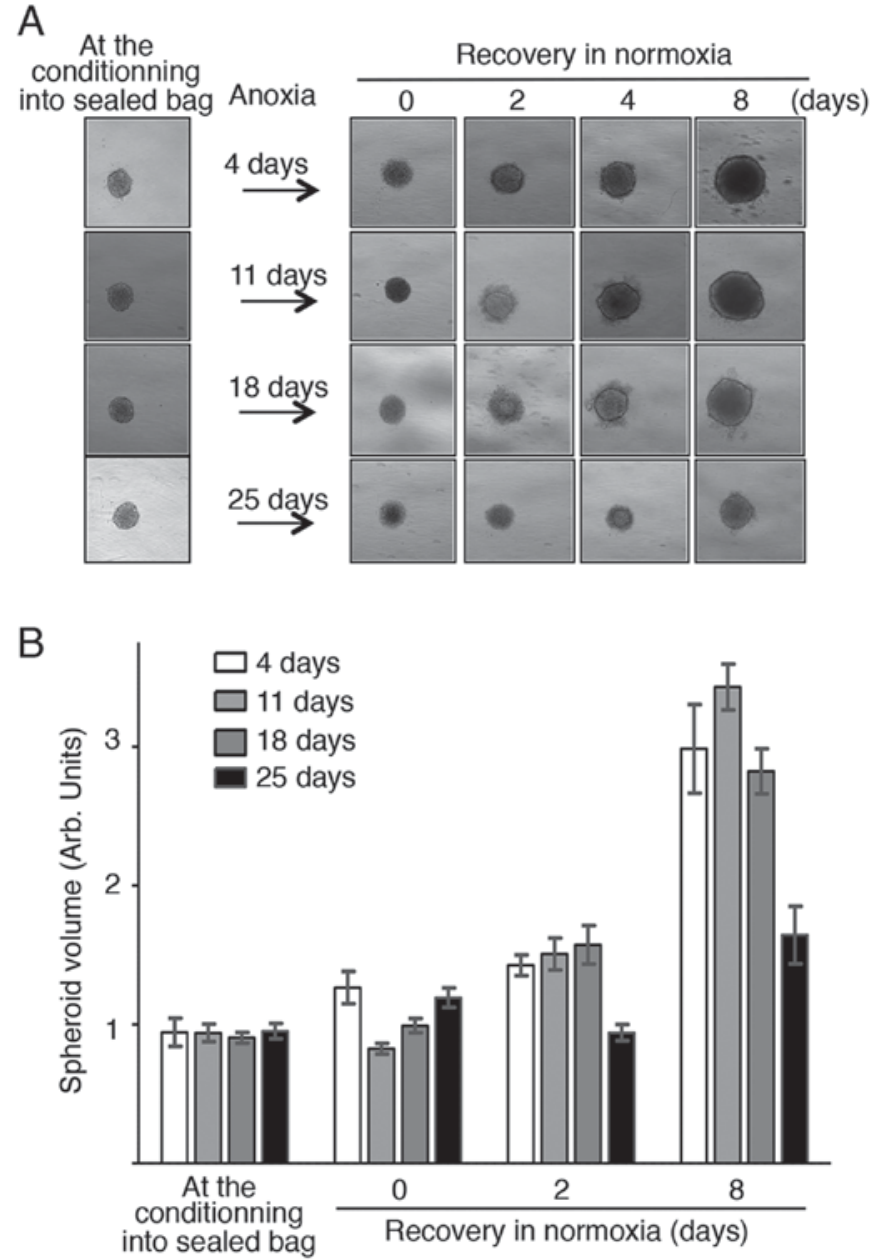

Figure 3. Reversible growth arrest in oxygen absorber-induced anoxia at $4^{\circ} \mathrm{C}$. (A) Representative micrographs illustrating the effect of anoxia at $4^{\circ} \mathrm{C}$ for $4,11,18$ and 25 days on spheroid growth and during recovery at $37^{\circ} \mathrm{C}$ in normoxia. (B) Spheroid volume determination before (at packaging), after anoxic storage at $4^{\circ} \mathrm{C}$ for $4,11,18$ and 25 days (0) and during recovery. Data are the mean $( \pm \mathrm{SD})$ of 50-60 spheroids per determination.

but not for 11 days (Fig. 2B). Indeed, spheroids kept in anoxic conditions for 11 days, rapidly dissociated and did not resume growth upon return to normoxia (Fig. 2B, lower panels). Quantification of the spheroid volume (60 spheroids/condition for each time point) confirmed that growth of spheroids stored at $37^{\circ} \mathrm{C}$ in anoxia for 4 days was totally inhibited during the storage period, but proliferation resumed once back to normoxia, with a slope similar to controls (Fig. 2C).

Together, these observations indicate that when placed at $37^{\circ} \mathrm{C}$ in an anoxic environment obtained with an ATCO Biosystem 96P oxygen absorber, spheroid proliferation is arrested. Back to normoxia, proliferation resumes in the same way as for control spheroids.

Spheroid growth is reversibly arrested by storage in oxygen absorber-induced anoxia at $4^{\circ} \mathrm{C}$ up to 18 days. Although it may be of interest to slow down or stop microtissue proliferation at $37^{\circ} \mathrm{C}$ for a short period, the storage and transport of such biological material would be more easily performed in standardized refrigerated conditions. Therefore, the ability of spheroids to resume proliferation after storage in oxygen absorber-induced anoxia at $4^{\circ} \mathrm{C}$ was assessed using 
the same experimental set-up (Fig. 2A). Plates were kept in anoxia, or not (controls), at $4^{\circ} \mathrm{C}$ for $4,11,18$ and 25 days, before returning to normoxia at $37^{\circ} \mathrm{C}$ for recovery. Similarly to the results obtained at $37^{\circ} \mathrm{C}$, oxygen absorber-induced anoxia led to growth arrest without loss of structure integrity or structure changes (Fig. 3A). Growth was resumed after return to normoxia, although it was less fast in spheroids stored in anoxia at $4^{\circ} \mathrm{C}$ for 18 and 25 days compared with up to 11 days. On the other hand, spheroids stored at $4^{\circ} \mathrm{C}$ without oxygen absorber could not resume growth after return to normal culture conditions (data not shown). Quantification of the spheroid volume (50-60 spheroids/condition per time point) at different time points after return to normoxia (Fig. 3B) confirmed growth resumption after storage at $4^{\circ} \mathrm{C}$ for up to 18 days.

These results indicate that storage of spheroids at $4^{\circ} \mathrm{C}$ in an anoxic environment obtained with an ATCO Biosystem 96P oxygen absorber leads to fully reversible growth arrest (up to 18 days of anoxia), which is technically very easy to achieve.

Spheroid storage in oxygen absorber-induced anoxia does not modify the response to etoposide. To definitively confirm that oxygen absorber-induced anoxia may represent a major advance for spheroid storage, spheroid preservation was evaluated by assessing their response to etoposide, a DNA polymerase inhibitor currently used in the clinic for cancer treatment. To this aim, spheroids stored with the oxygen absorber at $4^{\circ} \mathrm{C}$ for 4,7 or 14 days were allowed to recover in normoxia for $24 \mathrm{~h}$ before incubation with increasing concentrations of etoposide for $72 \mathrm{~h}$. Growth inhibition caused by etoposide cytotoxic effect was comparable in stored spheroids and in controls (spheroids of similar diameter at the time of treatment grown in normoxia), with similar $\mathrm{IC}_{50}$ values (Table I). Thus, storage of spheroids in anoxia at $4^{\circ} \mathrm{C}$ for 4,7 or 14 days does not modify their response to a reference genotoxic agent.

In this study, we investigate whether anoxia generated by using ATCO Biosystem $96 \mathrm{P}$ oxygen absorbers represents a valid method for the storage and shipment of 3D tumor spheroids. We found that by simply packing a 96-well plate in a heat-sealed plastic bag that contains an oxygen absorber, total anoxia can be generated in about $2 \mathrm{~h}$. Moreover, we show that in conditions of total anoxia, spheroid growth is fully stopped and that proliferation can be resumed after up to 4 days of storage in anoxia at $37^{\circ} \mathrm{C}$.

Mammalian cells are in principle unable to survive in hypoxic conditions because the unbalance between the decreasing ATP supply and the demand to ensure homeostasis progressively leads to mitochondria dysfunction and cell death. However, adaptive molecular responses allow a hypometabolic response that transiently prevents cell death (16). Recent work has shown that in acidic conditions, hypoxia can promote tumor cell survival by preserving the ATP level (17). Furthermore, a study on pancreatic islet conservation prior to transplantation demonstrated that storage at low temperatures prevent cell damage associated with hypoxia and may improve transplantation efficiency (18). In line with these reports, here we found that lowering the temperature to $4^{\circ} \mathrm{C}$ offers the possibility to fully resume spheroid growth after up to 18 days of anoxia. Although further work is needed to validate this storage method in other cancer cell lines our results already open a real and major opportunity for spheroid storage, functional preservation and shipment.

\section{Acknowledgements}

The support of the TRI-Genotoul and ITAV imaging facility is gratefully acknowledged. The authors would like to thank Elisabetta Andermarcher for expert manuscript editing. The work in the laboratory of B.D. and V.L. is supported by the University of Toulouse, the CNRS, Agence Nationale de la Recherche ANR and la Ligue Contre le Cancer.

\section{References}

1. Hirschhaeuser F, Menne H, Dittfeld C, West J, Mueller-Klieser W and Kunz-Schughart LA: Multicellular tumor spheroids: An underestimated tool is catching up again. J Biotechnol 148: 3-15, 2010.

2. Fennema E, Rivron N, Rouwkema J, van Blitterswijk C and de Boer J: Spheroid culture as a tool for creating 3D complex tissues. Trends Biotechnol 31: 108-115, 2013.

3. Ivascu A and Kubbies M: Rapid generation of single-tumor spheroids for high-throughput cell function and toxicity analysis. J Biomol Screen 11: 922-932, 2006.

4. Del Duca D, Werbowetski T and Del Maestro RF: Spheroid preparation from hanging drops: Characterization of a model of brain tumor invasion. J Neurooncol 67: 295-303, 2004.

5. Purcell WM, Atterwill CK and Xu J: Cryopreservation of organotypic brain spheroid cultures. Altern Lab Anim 31: 563-573, 2003.

6. Magalhaes R, Wang XW, Gouk SS, Lee KH, Ten CM, Yu H and Kuleshova LL: Vitrification successfully preserves hepatocyte spheroids. Cell Transplant 17: 813-828, 2008.

7. Sutherland RM, Sordat B, Bamat J, Gabbert H, Bourrat B and Mueller-Klieser W: Oxygenation and differentiation in multicellular spheroids of human colon carcinoma. Cancer Res 46: 5320-5329, 1986.

8. Kunz-Schughart LA, Freyer JP, Hofstaedter F and Ebner R: The use of 3-D cultures for high-throughput screening: The multicellular spheroid model. J Biomol Screen 9: 273-285, 2004.

9. Laurent J, Frongia C, Cazales M, Mondesert O, Ducommun B and Lobjois V: Multicellular tumor spheroid models to explore cell cycle checkpoints in 3D. BMC Cancer 13: 73, 2013.

10. Sutherland RM: Cell and environment interactions in tumor microregions: The multicell spheroid model. Science 240: $177-184,1988$.

11. Karlsson H, Fryknäs M, Larsson R and Nygren P: Loss of cancer drug activity in colon cancer HCT-116 cells during spheroid formation in a new 3-D spheroid cell culture system. Exp Cell Res 318: 1577-1585, 2012.

12. Gomes A, Guillaume L, Grimes DR, Fehrenbach J, Lobjois V and Ducommun B: Oxygen partial pressure is a rate-limiting parameter for cell proliferation in $3 \mathrm{D}$ spheroids grown in physioxic culture condition. PLoS One 11: e0161239, 2016.

13. Brody A, Strupinsky E and LR K: Active packaging for food applications. CRC Press, 2001.

14. Standa Industrie: Application de l'absorbeur d'oxygène ATCO dans les musées, bibliothèques et archives. La lettre de l'OCIM 60: 29-32, 1998 (In French).

15. Maekawa $\mathrm{S}$ and Elert K: The use of oxygen-free environments in the control of museum insect pests. The Gerry Conservation Institute, Los Angeles, 2003.

16. Boutilier RG: Mechanisms of cell survival in hypoxia and hypothermia. J Exp Biol 204: 3171-3181, 2001.

17. Parks SK, Mazure NM, Counillon L and Pouysségur J: Hypoxia promotes tumor cell survival in acidic conditions by preserving ATP levels. J Cell Physiol 228: 1854-1862, 2013.

18. Itoh T, Sugimoto K, Takita M, Shimoda M, Chujo D, SoRelle JA, Naziruddin B, Levy MF and Matsumoto S: Low temperature condition prevents hypoxia-induced islet cell damage and HMGB1 release in a mouse model. Cell Transplant 21: 1361-1370, 2012. 